\title{
The neurological practice of a district general hospital
}

\author{
J I MORROW, V H PATTERSON
}

From Downe Hospital, Downpatrick, and the Department of Neurology, Royal Victoria Hospital, Belfast, Northern Ireland.

SUMMARY In a one year prospective study, $19 \%$ of 925 adult medical admissions to a district general hospital (the Downe Hospital, Downpatrick, Northern Ireland) were due primarily to a neurological complaint. A further 19 cases had an active neurological disorder which contributed to their hospital admission. Most were acute admissions via the General Practitioner or through the Accident and Emergency Department, a situation different from the practice of most regional neurological centres. Only 15 patients ( $8 \%$ of the neurological cases) were referred to a specialist centre.

There has been much discussion recently on the role of neurologists and, indeed, of the different types of neurologist. ${ }^{1}$ A number of epidemiological surveys have been carried out, mostly in the United States ${ }^{2-4}$ where it has been estimated that $2.5 \%$ of the population each year require the attention of a physician competent in neurology. ${ }^{2}$ Little is known, however, about the numbers of patients with neurological problems who are seen in district general hospitals within the United Kingdom, about the nature of their problems, the treatment they receive and the criteria for referral to specialist centres practiced in these hospitals.

A study of these matters was undertaken in the hope that it might provide some useful information and yield some guidelines to the best use of neurological resources.

\section{The Health District and its Services}

Hospital services in the county town of Downpatrick date from 1767 with the establishment of an infirmary. The present Downe Hospital buildings date from about 1834 . The hospital serves a mainly rural population of about $53,000 .^{5}$ It is situated 22 miles from Belfast where all the regional specialities are to be found (see map) and it is the smallest Unit of Management in the Eastern Health and Social Services Board, which is responsible for a total population of 614,000 .

Primary health care in the district is the

Address for reprint requests: Dr J I Morrow, Epilepsy Unit, Department of Pharmacology and Therapeutics, University of Wales College of Medicine, Heath Park, Cardiff CF4 4XW, Wales, UK.

Received 18 November 1986. Accepted 2 April 1987.

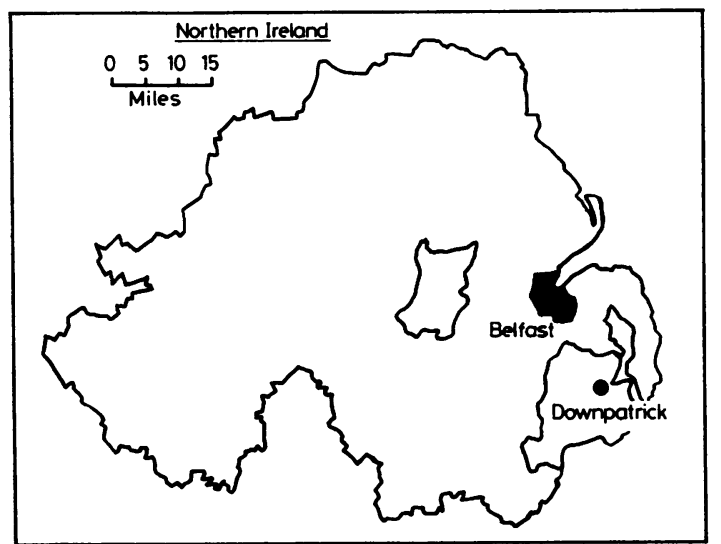

responsibility of 30 General Practitioners. The hospital has 104 beds and provides an Accident and Emergency service and both inpatient and outpatient services in general medicine, general surgery, gynaecology and ENT surgery. There are, in addition, outpatient clinics in chest medicine, dermatology, medical paediatrics, orthopaedic surgery, ophthalmology and radiotherapy, but there is no visiting neurologist. The services at the hospital are complemented by an obstetric unit and a geriatric medical assessment unit situated in the grounds of the neighbouring 893-bedded Downshire Psychiatric Hospital.

The medical unit of the hospital consists of 25 beds, five of which are devoted to coronary care. There are two consultant physicians with an on-call responsibility for general medicine, one of whom also has responsibility for the geriatric medical assessment 
Table 1 Number of neurological admissions by source

\begin{tabular}{llllll}
\hline & $G P$ & $A \& E$ & $O P$ & $D O M$ & DCCU \\
\hline $\begin{array}{l}\text { Number of admissions } \\
\begin{array}{l}\text { Percentage of total neurological } \\
\text { admissions }\end{array}\end{array}$ & 65 & 73 & 12 & 17 & 9 \\
\hline
\end{tabular}

Table 2 Neurological admissions by diagnosis

\begin{tabular}{lcc}
\hline & Number & $\begin{array}{l}\text { (\% of } \\
\text { neurological } \\
\text { cases) }\end{array}$ \\
Diagnosis & 46 & $26 \%$ \\
\hline Stroke & 26 & $14 \%$ \\
Epilepsies & 15 & $8 \%$ \\
Transient ischaemic attack & 11 & $6 \%$ \\
Dementia & 11 & $6 \%$ \\
Single tonic/clonic seizure & 11 & $6 \%$ \\
Blackout? cause & 7 & $4 \%$ \\
Severe headache & 6 & $3 \%$ \\
Migraine & 5 & $3 \%$ \\
Drug or alcohol induced & 4 & $2 \%$ \\
$\quad$ neurological syndrome & 4 & $2 \%$ \\
Meningitis & 4 & $2 \%$ \\
Subarachnoid haemorrhage & 3 & $2 \%$ \\
Cerebral tumour & 2 & $1 \%$ \\
Multiple sclerosis & 2 & $1 \%$ \\
Parkinson's disease & 2 & $1 \%$ \\
Post-Concussive syndrome & 2 & $1 \%$ \\
Radiculopathy & 2 & $1 \%$ \\
Vertebrobasilar insufficiency & 2 & $1 \%$ \\
Motor Neuron disease & 1 & $1 \%$ \\
Neuropathy & 14 & $8 \%$ \\
Encephalitis & 180 & \\
Neurological symptoms without & & \\
defined disease & & \\
Total & & \\
\hline
\end{tabular}

unit. This unit also accepts a small number of direct medical admissions but these patients were not included in the survey. The junior medical staff consisted of a registrar who had 6 months training in neurology, one senior house officer and one pre-registration house officer, night cover being shared with the junior staff from the geriatric assessment unit. The medical staff also had responsibility for the day-to-day care of any medical paediatric patients in the paediatric unit under the guidance of a visiting paediatrician. These paediatric cases were not included in the survey.

Patients could be admitted to the medical unit by a mobile coronary care unit (MCCU) and by transfer from the Downshire Psychiatric Hospital as well as by the usual referral sources from the General Practitioner, outpatient clinics, Accident and Emergency Department or following a consultant domiciliary visit.

\section{Method}

A prospective study was carried out for a period of one year, from August 1983 to July 1984, on all admissions to the medical unit of the Downe Hospital. One of us (JM) was medical registrar during the 12 month period (VP advised on the study but took no part in the management of patients) Admissions with neurological diagnoses were then selected for further study. These cases were classified by final diagnosis and whether this was the primary reason for admission or an active contributory factor. Personal information such as age and sex as well as referral source, length of stay, neurological investigations and final disposal were compiled on every case. Those patients referred to the Regional Neurological or Neurosurgical Unit were also listed.

\section{Results}

There were 925 admissions during the period August $1983-J u l y ~ 1984$. One hundred and eighty cases $(19 \%)$ were diagnosed as suffering from a neurological complaint which was the primary reason for their admission to hospital. There were a further 19 patients $(2 \%)$ who had an active neurological disorder which, although not the primary reason for admission, was felt to contribute to their hospitalisation.

\section{Sources of admission}

The majority $(77 \%)$ of patients were admitted either through the Accident and Emergency Department (A \& E) or at the request of the General Practitioner (GP). A much smaller number of cases were admitted from outpatients (OP), domiciliary visits (DOM) or by transfer from the Downshire Psychiatric Hospital. A further four patients were admitted by the mobile coronary care unit (MCCU). (Table 1 summarises sources of admission).

\section{Primary neurological diagnoses}

These are summarised in table 2 . As might have been expected vascular disease formed the largest category, with stroke the single most common neurological disorder seen ( $26 \%$ of all neurological cases). The average age of the 46 cases of stroke was 69 years (range 37-90 years) and also as might have been predicted they were among those that tended to stay in hospital the longest (average stay 24.0 days). Nine patients $(20 \%)$ were aged under 60 years. In four cases the stroke was felt to be in the vertebrobasilar territory, the rest being in the carotid territory.

Of these patients, 17 went home, 13 died, five had a period of rehabilitation in the geriatric unit, eight required some form of continuing care and three others were transferred to another hospital. 
Transient ischaemir attack (TIA) was the diagnosis in 15 patients. One presented with amaurosis fugax and 14 others had symptoms or signs which suggested another carotid artery disturbance. Two further cases were diagnosed as vertebrobasilar ischaemia. The TIA group tended to be younger (average age 63 years) as against those patients with completed stroke. Three of the patients had a completed stroke within the study year.

Subarachnoid haemorrhage was proved in four patients, three of whom were transferred to the Regional Neurosurgical Unit in Belfast. One man was not considered to be a surgical candidate and subsequently died in the Downe.

Epilepsy was the second most common diagnosis, there being 26 patients (14\%) in this group. Only three cases were admitted electively for stabilisation, observation or change of therapy. All the other cases were admitted after seizures and four were in status epilepticus. Fifteen patients had been previously diagnosed but in 11 the diagnosis was made for the first time. There were a further 11 cases who had had an isolated tonic/clonic seizure. These patients were not started on anticonvulsants but were followed up.

There were another 11 patients who had an episode of altered consciousness which was either not witnessed or of uncertain nature. If no diagnosis was reached they were labelled "blackout ? cause".

Acute confusional states were not included in the survey as the underlying diagnosis when ascertained was used for classification. There were, however, a number of patients with a chronic confusional state admitted for assessment, often after a recent deterioration in their mental state, although at least four cases were admitted partially on social grounds; all were clinically felt to be suffering from dementia. Four of the 11 cases were aged under 65 years.

A number of patients were admitted for investigation of headaches; all were admitted via the Accident and Emergency department or their GP. In all the aim was to exclude intracranial pathology and most had investigations carried out. In six the diagnosis was thought to be migraine. In seven others no firm diagnosis was reached but serious pathology had been excluded.

There were four cases of meningitis-one viral, one leptospiral, one meningococcal and one pneumococcal. There was also one case of viral encephalitis.

Multiple sclerosis was diagnosed in two patients, one possible and one probable. ${ }^{6}$ A further case of previously diagnosed multiple sclerosis was admitted with a relapse of her condition.

Motor neuron disease was diagnosed in one patient while another with the disease was admitted for terminal care.

Of the patients with cerebral tumours, two had metastases, one a primary pituitary tumour and another a recurrence of a chromophobe adenoma.

In the drug and alcohol group, two were admitted with ataxia and falls due in one case to chronic alcoholism and the other to phenytoin toxicity. The other three patients all came from the nearby psychiatric hospital and all had adverse reactions to neuroleptic drugs. Two had severe extrapyramidal syndromes and the other the neuroleptic malignant syndrome.

\section{Secondary neurological diagnoses}

There were a further 19 cases whose reason for admission, although not primarily neurological, was due at least in part to an underlying neurological disorder. In this category the largest group was of patients with immobility due to chronic neurological disability who had developed a chest infection. The second largest group was of peripheral neuropathy detected usually in diabetic patients with leg ulcers. Epileptic seizures occurred in three cases admitted for unrelated reasons and one patient had a transient ischaemic attack while undergoing investigation for ischaemic heart disease.

\section{Neurological investigations}

Only plain radiology and lumbar puncture were available for the investigation of neurological disease within the Downe hospital. EEG, isotope brainscans and digital subtraction angiograms could be ordered in the main Belfast hospitals without transferring patient care. CT scanning was available but usually only in consultation with the Regional Neurological or Neurosurgical Units in the case of suspected neurological disease and this usually meant transferring patient care. In only two cases was a CT scan performed and the patient returned to Downpatrick. Investigations that were carried out after transfer of the patient were not included. Table 3 summarises these figures.

\section{Referrals}

Of the 180 neurological cases seen in the hospital, 15 $(8 \%)$ were referred to the Regional Neurological or Neurosurgical Units, eight to a neurosurgeon and seven to a neurologist. The slight disproportion in favour of the surgeons was because it was simpler in practical terms to arrange a CT scan through that unit.

Those transferred to the Neurosurgical Unit

Table 3 Investigations

\begin{tabular}{llllll}
\hline & $L P$ & $E E G$ & ISOTOPE SCAN & CT SCAN & DSA \\
\hline Total & 29 & 31 & 26 & 2 & 2 \\
\hline
\end{tabular}

DSA = Digital Subtraction Angiography 
Table 4 Outcome

\begin{tabular}{lrr}
\hline & No & $\%$ \\
\hline Discharged & 122 & $68 \%$ \\
Died & 21 & $12 \%$ \\
Referred to neurological/neurosurgical unit & 15 & $8 \%$ \\
Long term hospitalisation & 12 & $7 \%$ \\
Other transfers & 10 & $6 \%$ \\
\hline
\end{tabular}

included three cases of subarachnoid haemorrhage, four cases of stroke, all of whom were relatively young and also one case of meningitis because of a history of recent head injury.

Because of difficulty in controlling the seizures of one patient admitted in status epilepticus, she was transferred to the care of a neurologist. Also referred as inpatients were individual cases of headache, cerebral tumour and one patient with a suspected spinal lesion. Both new cases of multiple sclerosis were referred to a neurologist as outpatients as was one case of severe drug-induced Parkinson's disease.

\section{Outcome}

Of the original 180 neurological cases admitted, 122 $(68 \%)$ were discharged, $21(12 \%)$ died, $25(14 \%)$ were transferred to another hospital or retransferred to the Downshire Psychiatric Hospital and $12(7 \%)$ required long term, usually geriatric, accommodation.

\section{Discussion}

The results that we have obtained give an indication of the neurological workload in a district general hospital where there is no resident or visiting neurologist and where patients with neurological disease are looked after by a general physician. District general hospitals are of course not all the same because of local variables and practices. The Downe Hospital for example has a relatively small catchment population.

The results show firstly that neurological illness is a common cause of medical admission to hospital, accounting for about $20 \%$ of cases admitted. Secondly most neurological cases are acute admissions admitted either through the Accident and Emergency department or at the request of the General Practitioner. This is quite different from the practice of neurology in many neurological units where the majority of admissions are elective. Thirdly the pattern of neurological investigation is quite different from that at a neurological or neurosurgical centre and probably reflects the lack of availability of CT scanning in particular. Although it is not possible to comment on the propriety of investigation except on an individual basis, there does seem to be an excess of lumbar puncture and isotope scans. Fourthly, only a small percentage of patients with neurological illness seen primarily at a district general hospital are referred to a regional centre. This is in marked contrast to the situation in the USA where in calculating future requirements for neurologists ${ }^{7}$ it was expected that high percentages of common neurological complaints should be seen by a neurologist. This seems to highlight the considerable differences in terms of patient expectation and availability of neurologists between the UK and the United States.

One would expect a physician trained as a neurologist to manage patients with neurological disease more efficiently and effectively than a physician with little neurological training. If this is true then the implication that can be drawn from this study is that patients with acute neurological disease in the UK are not at present receiving the most efficient and effective management.

How then can this situation be improved? Firstly, should neurology figure more in postgraduate training of a general physician? Given that $20 \%$ of the admissions are neurological, 12 months at a registrar or senior registrar level would not seem excessive. At present there is no stipulation or even recommendation from the Royal Colleges of Physicians on this subject. This would certainly increase awareness of neurological practice by physicians although it might not necessarily expose them to the type of neurology seen in district general hospitals.

Would an increase in visits from a neurologist help? One or more weekly visits from a neurologist would enable not only new and review outpatients to be seen locally but also neurological inpatients to be seen at the request of the local physicians. If a good working relationship was established then the practices of the neurologist should positively influence the neurological practice of the physicians. Such contact would also establish a personal line of communication to the Regional Neurological Centre and should make the ordering of specialised investigations easier. Thus acute neurological patients would continue to be managed by physicians who would have much more readily available neurological advice. One session per week from a neurologist would cover a population of about 50,000 people and with three such weekly clinics this would mean one neurologist per 150,000 of the population. This arrangement would require a doubling of the present numbers of consultant neurologists.

Should a resident neurologist be attached to every district general hospital? In a comparable catchment population of 53,000 in the United States there would be 1.5 neurologists and probably one CT scanner; in Norway there would be one neurologist. For comparable levels to be achieved in the UK the number of neurologists would have to be increased about 10 fold and this is probably unrealistic. 
neurology be created for every district general hospital? There has been discussion of this lately but little thought has been given to how such a person should be trained. Would they be sufficiently competent after one year's neurological training or would they need two or more? The type of neurology seen at neurological "centres of excellence" would be very different from that of a district general hospital and so would they need a different type of neurological training? The figures would suggest that one-fifth of all consultant physicians appointed should be of this variety. Its creation would clearly have far reaching implications for medical and neurological training in the UK.

These are only some possible solutions. They are not necessarily mutually exclusive and there may be others. We believe that the care of patients with acute neurological disease in the UK can and should be improved and the first step might be a reappraisal of the role of the neurologist.

Our thanks to Dr. F. McAleenan and Dr. A. J. Cameron for their kind co-operation in the compilation of this paper. Our thanks also to Professor J. A. Aarli, for figures of neurologist numbers in Norway.
This paper has been presented in part to the meeting of the Association of British Neurologists with the American Neurological Association at the London Hospital, 1st-2nd November 1985.

\section{References}

1 Hopkins A. Different types of neurologist. $\mathrm{Br}$ Med J 1984;288:1733-6.

2 Kurtzke JF. The current neurologic burden of illness and injury in the United States. Neurology 1982;32: 1207-14.

3 Kurtzke JF. Neuroepidemiology. Ann Neurol 1984;16: 265-77.

4 Guez M, Anderson PJ. Frequency of neurologic diagnoses: Implications for curriculum design. Mt Sinai $J$ of Med 1980;47:364-72.

5 Ninth Annual Report of the Eastern Health and Social Services Board, Northern Ireland 1983.

6 McDonald WI, Halliday AM. Diagnosis and classification of multiple sclerosis. $\mathrm{Br}$ Med Bull 1977;33:4-8.

7 Garrison LP, Bowman MA, Perrin EB. Estimating physician requirements for neurology: A needs based approach. Neurology 1984;34:1218-27. 